\title{
Aspects économiques et réglementaires liés à la circulation du poisson
}

\author{
Economic and rule-governing aspects \\ linked to the circulation of fish
}

\author{
S. Martin J.-P. Tane
}

Ministère de l'Environnement

Direction de la protection de la nature

Service de la pêche et de l'hydrobiologie

92524 Neuilly-sur-Seine

Les auteurs rappellent l'importance économique et sociale de la pêche fluviale et maritime ainsi que le déficit global de la balance commerciale de la pêche en France. Un effort de restauration a été fait au cours de la dernière décennie pour arrêter la chute des effectifs de certaines espèces amphihalines (saumons, esturgeons). Dans une deuxième partie est évoqué le cadre réglementaire d'interventions prenant appui sur les dispositions de la loi du 10 juillet 1976 sur la protection de la nature et du 29 juin 1984 sur la pêche en eau douce et la gestion des ressources piscicoles (article 411 CR notamment). La libre circulation du poisson est un élément fondamental du maintien des cycles biologiques et de la diversité des écosystèmes. Elle concerne non seulement les grands migrateurs amphihalins, mais aussi les espèces sténohalines (truites, brochets, etc.). Les nouvelles dispositions prévoient une obligation de résultat et la distinction entre pêcheurs professionnels et amateurs. La participation des organismes aménageurs est naturellement impérative pour la réussite des programmes de restauration. Les mesures s'appliquent aux constructeurs de barrages de microcentrales, de seuils, de dispositifs de prises et de rejets d'eau. Cette participation peut prendre la forme de conventions dans le cadre de contrats Etat-Régions, etc.

The writers refer to the economic and social importance of fresh-water and deep-sea fishing as well as the overall deficit of the trade balance of French fishing. During the last decade, an effort has been made to reduce the disappearance of certain " amphihaline "* species (salmon, sturgeons). The second part links the statutory rules concerning human interventions and relating to the provisions of the Act of 10th July 1976 concerning protection of the natural environment and the Act of 29 June 1984 concerning fresh-water fishing the management of piscicultural resources (article $411 \mathrm{CR}$ in particular). The free circulation of fish is a fundamental element in maintaining biological cycles and the diversity of ecosystems. It concerns the large migrating "amphihaline "* as well as the stenohaline species (trout, pikes, etc.). The new provisions provide for a resultant obligation and the distinction between professional fishermen and amateurs. The participation of improvement bodies is vitally needed for the success of restoration programmes. The steps to be taken apply to builders of dams, dikes, water inlet and outlet devices. This participation can take the form of agreements within the framework of State/Departmental contracts, etc.

* "amphihaline \# : a sub-family of salmon, trout species easily adaptable to fresh water and salt water. 


\subsection{Préambule}

De tous temps, l'homme s'est intéressé à la vie de la rivière. Ses premiers pas ont été difficiles et sa survie a été liée pendant des siècles à la nourriture qu'il pouvait récolter dans le milieu naturel.

De nos jours, l'eau, le milieu naturel aquatique, le poisson, continuent à exercer une certaine fascination sur nos contemporains. La pêche sportive est née progressivement et a pris peu à peu une importance économique et sociale de premier plan.

\subsection{Importance économique et sociale de la pêche en France}

L'activité la pêche était autrefois un moyen de se procurer des protéines. Il y a quelques années encore, l'obligation de "faire maigre" le vendredi entraînait une consommation de poisson d'eau douce importante. L'apparition de nouvelles techniques de conditionnement a élargi la gamme des poissons offerte aux consommateurs à l'état frais, congelés ou surgelés. La balance française du commerce extérieur des produits de la pêche* est déficitaire depuis de nombreuses années en dépit du domaine maritime très étendu dont nous disposons. Les exigences du consommateur s'orientent vers des poissons considérés comme noble tels le saumon, la sole, la daurade, etc. Au niveau des eaux douces, le développement industriel a entraîné une dégradation du milieu naturel qui a coïncidé avec le changement des habitudes alimentaires évoquées ci-dessus. La pêche professionnelle en eau douce** a perdu de son importance économique et la pêche professionnelle en zone maritime connaît des jours difficiles (raréfaction du poissons, coût énergétique de plus en plus élevé pour obtenir une même récolte, difficultés de commercialisation de certaines espèces de poissons).

A l'inverse, la pêche sportive en mer et surtout sur les eaux intérieures a connu un développement foudroyant depuis le début du siècle. Le pêcheur sportif, rendu plus mobile par les progrès des moyens de locomotion, est devenu un acteur économique important. Cette pêche sportive est liée à un tourisme du bord de l'eau que des enquêtes récentes permettent de mieux cerner. En France, près de deux pêcheurs sur trois sur un total de quatre millions de pratiquants ou assimilés, consacrent plus de trente jours par an à ce loisir. Une évaluation établie en 1982***, fixée à quatre milliards de francs, les retombées économiques de la pêche mesurées en terme de revenus associés à l'hébergement des pêcheurs et de leur famille. L'achat de matériels et d'équipements de pêche correspondait à la même date à un montant de un milliard de francs.

\subsection{Programmes de soutien de l'activité pêche et défense du milieu naturel aquatique}

Pour accompagner "la montée en puissance " du tourisme pêche, et dans le contexte général d'une meilleure prise en compte de son environnement, des programmes de soutien de l'activité pêche et de défense du milieu naturel aquatique ont été lancés depuis ces vingt dernières années. Citons notamment :

- le programme Saumon 1976-1980 approuvé par décision du CIANE du 30 juillet 1975 ;

- le plan poissons migrateurs 1981-1982 a élargi les interventions aux espèces amphihalines (esturgeon, lamproies, alose et truite de mer);

- le plan quinquennal de mise en valeur des milieux aquatiques 1982-1986 a étendu les interventions à l'ensemble du milieu des eaux douces et a notamment mis en œuvre une relance du tourisme pêche.

\subsection{Adaptation à l'évolution économique récente}

L'évolution décrite ci-dessus traduit une prise de conscience du public et des dirigeants de la pêche de la nécessité d'une reconquête du milieu naturel remarquable que constituent les eaux douces. Cette reconquête a été facilitée par la mise en place, depuis une vingtaine d'années, de mesures visant à réduire la pollution des eaux et à restaurer la qualité des cours d'eau français.

Le réseau hydrographique français, correspondant à deux cent soixante quinze mille kilomètres de rivières, dont quinze mille kilomètres de cours d'eau navigables et de canaux, offre un potentiel de grande valeur. Le ralentissement de l'activité économique intervenu, dans la dernière décennie, a incité la société à se replier sur elle-même et chercher une expansion économique sur le marché intérieur. Ce contexte a certainement une influence positive sur la relance d'un tourisme lié à une redécouverte des richesses naturelles de l'hexagone.

\subsection{Cadre législatif et réglementaire}

Pour accompagner cette reconquête du milieu intérieur, une loi cadre a été mise en place après dix ans de réflexion et de maturation. Elle a pris la forme d'une loi sur la pêche en eau douce et la gestion des ressources piscicoles. Cette loi adoptée à l'unanimité, promulguée le 29 juin 1984 traduit l'évolution du législateur dans ce domaine et illustre la prise de conscience générale :

Article $\mathrm{I}^{\mathrm{tr}}$ - «La préservation des milieux quatiques et la protection du patrimoine piscicole sont d'intérêt général - La protection du patrimoine piscicole implique une gestion équilibrée des ressources piscicoles dont la

* Se reporter notamment à « La production mondiale de saumons 1952-1982 et le commerce extérieur français de saumons (1956-1983) - Thibault, Boude, J.P. Prevost, E., INRA - ENSA, Ecologie hydrobiologique, Economie halieutique - 35042 Rennes Cedex" (décembre 1984).

** La pêche professionnelle en eau douce représentait en 1982 un tonnage approximatif de 2000 tonnes pour une valeur de 50 millions de francs.

*** Se reporter à l'étude «Impact Economique de la pêche de loisir en eau douce. IDET-CEGOS 1982 " - in "Données Economiques de l'Environnement " - La Documentation Française. 
pêche, activité à caractère social et économique constitue le principal élément ".

Cet énoncé est à rapprocher des dispositions prévues par la loi du 10 juillet 1976 relative à la protection de la nature, qui dans son article $1^{\text {er }}$ stipule :

Article $1^{e r}-$ " La protection des espèces naturelles et des paysages, la préservation des espèces animales et végétales, le maintien des équilibres biologiques auxquels ils participent et la protection des ressources naturelles contre toutes les causes de dégradation qui les menacent sont d'intérêt général ".

La loi sur la pêche est donc venue renforcer le dispositif législatif et préciser, pour les milieux constitués par les eaux douces, le cadre de protection réglementaire précédemment élaboré.

\subsubsection{Libre circulation du poisson}

\section{a) Dans le bras courtcircuité}

L'article 410 indique que " tout ouvrage à construire dans le lit d'un cours d'eau doit comporter des dispositifs maintenant dans ce lit un débit minimal garantissant en permanence la vie, la circulation et la reproduction des espèces qui peuplent les eaux au moment de l'installation de l'ouvrage, etc." .

\section{b) Circulation du poisson à travers l'ouvrage}

L'article 411 précise que "dans les cours d'eau ou parties de cours d'eau et canaux... tout ouvrage doit comporter des dispositifs assurant la circulation des poissons migrateurs. L'exploitant de l'ouvrage est tenu d'assurer le fonctionnement et l'entretien de ces dispositifs.

"Les ouvrages existants doivent être mis en conformité sans indemnité avec les dispositions du présent article dans un délai de cinq ans à compter de la publication d'une liste d'espèces migratrices par bassin ou sous-bassin fixée par le ministre chargé de la pêche en eau douce et, le cas échéant, par le ministre chargé de la mer " (arrêté ministériel du 2 janvier 1986 - J.O. du 4 février 1986 pages 1959 à 1966).

L'article 12 de la même loi prévoit que « le classement des cours d'eau, parties de cours d'eau et canaux intervenu en application de l'article $428-2^{\circ}$ du code rural antérieurement à l'entrée en vigueur de la présente loi vaut classement au titre de l'article 411 du même code ".

Il convient de rappeler que l'article $428-2^{\circ}$ est issu des dispositions de la loi du 31 mai 1865 qui prévoyait déjà la libre circulation du poisson.

On admirera, à cette occasion, la continuité de pensée du législateur et ses nobles intentions. Etaient parus avant l'adoption des nouvelles dispositions de 1976 et 1984 les décrets suivants :

- Bassin de la Seine - décret du 3 août 1904.

Bassin de la Loire - décret du $1^{\text {er }}$ avril 1905.

Bassin de la Canche - décret du 3 février 1921.

Bassin de l'Adour - décret du 15 avril 1921.

Cours d'eau bretons - décret du 31 janvier 1922.

Bassin de l'Authie - décret du 2 février 1922.

Cours d'eau normands - décret du 23 février 1924.
Manquaient à l'appel les bassins de la Garonne, de la Dordogne, du Rhône et les fleuves de l'Est de la France, tels le Rhin, la Meuse, la Moselle, etc.

L'instruction ministérielle PN-SPH $n^{\circ} 85 / 3250$ du 27 novembre 1985 définit les conditions d'application de l'article 411 . Elle s'appuie notamment sur un inventaire des ouvrages concernés par ces mesures.

Il convient toutefois d'insister sur le fait que l'obligation mise en place d'une échelle à poissons peut résulter non seulement des dispositions de l'article 411, mais aussi des dispositions réglementaires introduites dans le règlement d'eau de l'ouvrage à construire dans le cadre de son autorisation ou de sa concession, en application des dispositions générales de la loi sur la protection de la nature en vue de maintenir la diversité des écosystèmes.

\subsubsection{Maintien des cycles biologiques et de la diversité des écosystèmes}

Le ministère de l'environnement a publié depuis de nombreuses années, en application de la loi sur la protection de la nature, une série de textes visant à préserver les espèces menacées et protéger leurs habitats d'une dégradation irréversible.

En ce qui concerne le milieu aquatique sont à signaler notamment :

- l'arrêté du 25 janvier 1982 (J.O. du 14 février 1982) qui assure la protection intégrale de l'espèce "Acipenser sturio ". L'esturgeon européen, autrefois représenté dans les principaux axes fluviaux (Tamise, Seine, Rhin, Rhône, Loire) n'est plus observé que sur le bassin de la Garonne;

- l'arrêté du 12 février 1982 (J.O. du 24 février 1982) qui interdit de détruire et d'enlever sciemment les œufs des espèces suivantes sur leurs zones de frai et protection des zones particulières à ces espèces: lamproie marine, la proie fluviatile, grande alose, alose feinte, saumon atlantique et truite de mer;

- l'arrêté du 4 octobre 1985 (J.O. du 27 octobre 1985) étend la possibilité de la conservation des biotopes nécessaires à l'alimentation à la reproduction, au repos ou à la survie de différentes espèces parmi lesquelles on peut citer la truite, l'ombre commun, le brochet, l'apron, etc.

La conservation des biotopes des espèces amphihalines (esturgeon, lamproies, alose, anguille, saumon, truite de mer, etc.) et celle des espèces sténohalines (ombre, brochet, etc.) est donc assurée par l'ensemble de ces arrêtés. Cette protection spécifique suppose, bien évidemment, un effort considérable de cartographie et d'inventaire des peuplements des zones de frayères potentielles et des zones d'alimentation des alevins, juvéniles et adultes. Pour atteindre cet objectif, le ministère de l'environnement a publié une instruction ministérielle relative à la protection des écosystèmes d'eaux courantes en date du 20 novembre 1981. Cette instruction décrit notamment les conditions minimales à remplir pour obtenir une bonne description de l'état initial du cours d'eau et rappelle la nécessité de couvrir le cycle biologique complet des principales espèces de poissons présentes dans le milieu inventorié. 


\subsection{Mise en æuvre d'une protection et d'une gestion cohérente des milieux aquatiques et plus particulièrement des populations pisci- coles}

La loi sur la pêche en eau douce et la gestion des ressources piscicoles du 29 juin 1984 prévoit la mise en place, dans chaque bassin hydrographique, d'une commission chargée de "proposer les orientations de protection et de gestion des milieux aquatiques du bassin (article 417). Ces orientations sont arrêtées par le ministre chargé de la pêche en eau douce ".

Les articles 415 et 416 prévoient l'élaboration des schémas départementaux de vocation piscicole en conformité aux orientations de bassin.

L'article 424 stipule par ailleurs que «l'exercice d'un droit de pêche emporte obligation de gestion des ressources piscicoles. Celle-ci comporte l'établissement d'un plan de gestion.

La libre circulation du poisson est un élément fondamental du maintien du cycle biologique et de la diversité des écosystèmes. Les textes visés ci-dessus et le cadre réglementaire qui en découlera permettront aux différents partenaires d'avoir une information sur les espèces et les milieux à protéger.

\subsection{Prise en compte de populations de poissons migrateurs}

La politique de restauration et de préservation des populations de poissons migrateurs sera arrêtée par le ministre de l'environnement au niveau de chaque bassin sur proposition des commissions de bassin dans la mesure où ce problème dépasse le cadre départemental.

Les schémas départementaux de vocation piscicole reprendront, dans le détail, ces orientations en les traduisant en programmes précis d'actions, accompagnés de propositions cohérentes de mesures réglementaires.

Pour les espèces vivant alternativement en eau douce et en eau salée, des décrets en Conseil d'Etat règlent pour la pêche en eau douce et pour la pêche maritime, d'une manière uniforme : « les mesures utiles à la reproduction, au développement, à la conservation et à la circulation de ces espèces " (article 436-3 $3^{\circ}$.

Une instruction ministérielle conjointe Environnement/Secrétariat d'Etat chargé de la mer, en date du 24 janvier 1986, met en place pour chaque estuaire un groupe de travail devant faire des propositions d'ensemble pour les espèces migratrices.

\subsection{Mise en place de dispositions contractuelles sur les principaux axes fluviaux}

Pour fonctionner de manière correcte un ouvrage de franchissement doit être conçu, alimenté en eau et entretenu de manière satisfaisante. Lorsqu'un aménageur intervient sur plusieurs ouvrages du même axe fluvial il sera intéressant d'établir des dispositions contractuelles pour synchroniser de manière adéquate les aménagements et dispositifs concernés. La convention EDF/Environnement, intervenue le $1^{\text {er }}$ juillet 1982, devra donc chaque année faire l'objet d'un échéancier précis d'intervention des travaux.

D'autre part, les observations et constats établis lors de la discussion des schémas départementaux de vocation piscicole devront se traduire par le souci constant pour les administrations de tutelle (Directions départementales de l'agriculture et de la forêt - Directions départementales de l'équipement), de rappeler à chaque exploitant ou pétitionnaire ses obligations lors de l'installation d'ouvrages nouveaux ou de la modification d'ouvrages anciens. Dans un grand nombre de cas des aménagements rustiques mis en place aux emplacements judicieux permettront de satisfaire à ces obligations.

En ce qui concerne les aménagements et les dispositifs qui ne découleraient pas d'obligations réglementaires, des processus conventionnels (contrats Etat/Régions) seront susceptibles d'apporter des solutions positives aux problèmes de migration.

\subsection{Conclusion générale}

La libre circulation du poisson permet de maintenir, pour l'ensemble des espèces concernées, les possibilités, d'alimentation des phases juvéniles et les possibilités de reproduction des adultes. La libre circulation des poissons constitue bien un élément fondamental de maintien des cycles biologiques et de la diversité des écosystèmes. Chaque axe fluvial, chaque rivière, chaque petit ruisseau est un lieu d'échanges et de déplacement des populations piscicoles. Un milieu naturel riche et diversifié ne peut exister que si ces possibilités de circulation sont assurées. L'exemple des rivières canalisées, entrecoupées de barrages infranchissables montre de manière éloquente l'appauvrissement auquel on peut aboutir dans des situations extrêmes - «quand le poisson meurt, l'homme est menacé " - des exemples récents l'ont encore montré. La Meuse, la Seine, le Rhône, etc. ont perdu leur diversité écologique et le coût de leur restauration sera élevé.

Les programmes de restauration en cours ont donné des résultats encourageants et ont montré que les processus de dégradation n'étaient pas irréversibles. Les aménageurs sont invités à participer de manière active à cette restauration. On a pu observer notamment au colloque franco-québécois de Bergerac qui s'est déroulé du 28 mai au $1^{\text {er }}$ juin 1985 , consacré à la restauration des rivières à saumon que le retour de ce poisson-symbole pouvait déclencher un processus de réflexion et de prise de conscience de la nécessité d'une gestion globale de la rivière à laquelle doivent participer tous les riverains. Les exemples étrangers vont dans le même sens. La restauration de la Tamise ou la restauration de la rivière Connecticut aux Etats-Unis, de la rivière Jacques Cartier au Québec ont montré que cette prise de conscience était partagée par différents pays développés qui, ayant trop sacrifié au développement industriel anarchique, redécouvrent des possibilités d'expansion économique en restaurant leurs rivières et leurs plans d'eau. 
M. Le Président remercie M. TANE pour le lien qu'il a établi entre les aspects économiques et règlementaires et ajoute qu'il est nécessaire d'établir une réflexion globale à ce propos.

En effet, on aborde là le lien entre les aspects sociaux et les aspects économiques. Il semble indispensable que des objectifs précis soient établis tant au niveau national qu'au niveau régional; sinon on risque d'avoir un bel éventail de lois et de règlements concernant la protection de la nature. Si l'on ne se demande pas pour quoi faire, on risque d'être en porte-à-faux par rapport à la réalité et l'on risque l'inefficacité.

Il ne faut pas oublier les aspects économiques indirects dont vous avez montré l'intérêt. Il y a ceux auxquels on ne pense pas toujours. Par exemple, sur le plan de la santé, un aspect indirect pourrait être la prévention de certaines maladies, comme les maladies cardiovasculaires ou les maladies cancéreuses. On sait que, dans les pays à forte consommation de poisson, ces deux types de maladies existent peu ou sont beaucoup moins développées. Par conséquent, si, pour une population nationale, ou régionale, on rétablit un lien entre loisirs, économie des loisirs, habitudes alimentaires et apprentissage d'une consommation plus importante de poisson, on pourra espérer une diminution du taux de développement des maladies cardiovasculaires et cancéreuses.

Cet aspect semble anodin mais peut avoir un impact économique non négligeable.

Il faut s'interroger également sur la façon dont ce problème sera intégré aux objectifs de protection de la nature.

M. VENDROT signale que les pagayeurs sont amenés à utiliser les passes à poissons comme glissières afin que les bateaux puissent descendre la rivière sur sa totalité. Il demande si le plan de mise en valeur aquatique comporte un volet tourisme nautique.

$M$. TANE répond que le schéma départemental touche l'ensemble du milieu aquatique et devrait intégrer à ce niveau la prise en compte d'un tourisme lié à la découverte de la rivière, notamment pour le canoë-kayak. Reste à régler le problème d'entente entre les pêcheurs et pratiquants de canoë-kayak, mais on peut penser que leurs intérêts étant communs, dans beaucoup de cas, la prise en compte de cette sorte de tourisme peut être envisagée selon des modalités à définir.

Il serait intéressant de mieux cerner l'importance économique du développement de ce tourisme canoë-kayak.

$M$. VENDROT ajoute qu'une enquête vient d'être rédigée qui comporte un volet socio-économique.

M. LEGRAND s'interroge sur la contradiction apparente que comporte la loi Pêche, entre la création de groupements de pêcheurs amateurs autorisés à pratiquer la pêche aux engins et l'existence de pêcheurs professionnels.

Pour $M$. TANE la pêche aux engins amateurs correspond, dans certains secteurs, à une activité traditionnelle débouchant uniquement sur une consommation familiale et non pas sur une commercialisation éventuelle.

La loi Pêche est claire sur ce sujet : elle stipule que seuls les pêcheurs professionnels peuvent commercialiser.

L'objectif du groupe de travail mis en place a été de veiller à ce que les engins de pêche de chaque catégorie soient adaptés à leurs activités.

Le problème est assez délicat car il est parfois difficile de faire le partage entre les deux. Les décrets d'application de la loi viennent de sortir et devraient permettre une meilleure définition des obligations de chacune des parties.

M. ROGUET apporte les précisions suivantes sur les aspects économiques concernant la pêche professionnelle :

Nous avons des chiffres notamment pour l'estuaire de la Gironde et pour le bassin de la Garonne et de la Dordogne. Il a été mis en place de nouvelles associations de pêcheurs professionnels qui se distinguent des amateurs. On connaît le nombre des professionnels authentiques : nous en avons actuellement 390 pour le bassin de la Garonne et de la Dordogne (c'est le plus important groupement de France) et il y en a environ 310 qui pêchent dans l'estuaire.
La valeur économique des captures, en estuaire de la Gironde seulement, de grandes aloses, de lamproies marines, d'anguilles, de civelles et de salmonidés - saumons et truites de mer -, est de 1827 tonnes, pour une valeur de 46 millions de francs, soit un revenu de $150000 \mathrm{~F}$ par famille, ce qui prouve que ces pêcheurs peuvent vivre du produit de leur pêche.

Il faut ajouter que si certains projets de barrages qui ont été proposés à l'aval de la Garonne étaient autorisés, il y aurait bien des chances de rayer complètement ou partiellement ces revenus, auquel cas cela serait une catastrophe économique. Je dois dire que nous avons besoin de tels chiffres pour défendre nos positions. Il est dommage qu'on n'ait pas avancé des chiffres plus précis en ce qui concerne les autres aspects économiques de la pềche.

$M$. LUQUET pose deux questions, l'une à caractère réglementaire, l'autre à caractère économique.

La première est de savoir si l'on ne pourrait pas imposer systématiquement, dans le cadre de l'arrêté préfectoral d'autorisation des travaux pour l'utilisation de l'eau, la rédaction, par le pétitionnaire, d'un rapport concernant l'efficacité de ces passes à poissons. Ce rapport serait à fournir dans un délai défini.

La deuxième question est de savoir si l'on a actuellement (notamment à disposition des pétitionnaires) une idée du coût d'une passe à poissons en fonction du type de barrage.

Concernant l'aspect réglementaire, $M$. TANE précise que pour les ouvrages nouveaux, il n'y a pas de problème, le décret prévoyant le fonctionnement, l'entretien et l'efficacité de la passe à poissons.

Le problème reste posé pour les ouvrages anciens pour vérifier l'efficacité de la passe.

Il n'est pas certain qu'un rapport fait par le pétitionnaire suffise; il pense qu'un contrôle effectué par les agents chargés de la police des eaux pourrait s'avérer plus efficace dans les cas difficiles ou de conflits.

Concernant l'aspect coût, M. LARINIER indique qu'il a effectué une étude statistique sur une centaine d'ouvrages. Il est difficile de tirer des règles générales, compte tenu du nombre de paramètres intervenant dans le coût d'une passe à poissons (fait que le barrage existe ou non lors de la construction de la passe, plus ou moins grandes facilités d'accès au site, facteurs locaux ou régionaux, etc.).

La hauteur de lobstacle et le débit moyen annuel du cours d'eau sont les deux paramètres expliquant le mieux la variabilité du coût des dispositifs de franchissement, ce qui est logique dans la mesure où ces dispositifs sont généralement à l'échelle de l'obstacle et du cours d'eau.

Pour donner des ordres de grandeur, le coût des passes à ralentisseurs installées sur les petits cours d'eau à saumons bretons (chutes : 1,5 à $2,5 \mathrm{~m}$ ) varie de 100 à $200 \mathrm{kF}$. Dès que l'on passe à des cours d'eau plus importants (module de plusieurs dizaines de $\mathrm{m}^{3} / \mathrm{s}$ ), on arrive à des coûts de 500 à $600 \mathrm{kF}$ pour des chutes de 3-4 mètres. Sur les grands cours d'eau (module de plusieurs centaines de $\mathrm{m}^{3} / \mathrm{s}$ ), les coûts peuvent dépasser le million de francs. Exceptionnellement, cas de barrages existants et de migrateurs exigeants comme l'alose, le coût peut dépasser la dizaine de millions de francs : on citera pour mémoire la passe au barrage de Bergerac sur la Dordogne (11 $000 \mathrm{kF}$ ) et l'ascenseur de Golfech sur la Garonne (environ $15000 \mathrm{kF}$ ).

M. PICARD s'interroge sur les deux aspects suivants :

a) si le législateur, dans sa sagesse, a effectivement prévu l'obligation de résultats, c'est qu'en toute conscience il a estimé qu'il avait à sa disposition les moyens de contrôler l'efficacité des ouvrages;

b) il y a obligation systématique de prévoir des dispositifs de passage des poissons pour tout ouvrage. Cela pose un problème économique. Est-ce qu'au-delà d'un certain coût, notamment pour les migrateurs locaux, ne vaut-il pas mieux prévoir autre chose qu'une passe à poissons qui servira peu ou pas du tout ? Dans certains types d'ouvrages, on peut faire des aménagements qui sont compensateurs puisque les textes prévoient la compensation.

Peut-on échapper à cette obligation lorsqu'elle constitue une hérésie économique? 
Pour la première question, M. TANE répond qu'effectivement le législateur a tout prévu; il suffit de se donner les moyens de vérifier l'efficacité de l'ouvrage.

Dans les ouvrages nouveaux, le règlement d'eau le prévoit, le contrôle de l'efficacité se fait par des sondages. S'il y a un problème, les partenaires concernés par cet aspect comme les associations de protection de la nature..., peuvent demander une conférence administrative et s'il y a impossibilité technique le règlement d'eau interviendra.

Le contrôle de l'efficacité est tout à fait possible et il faut le mettre en cuvre.

Quand au deuxième aspect de l'ẻventuelle hérésie économique qui consisterait à construire une passe qui ne serait pas efficace, M. TANE estime qu'il faut être très réservé et que la question doit se discuter au cas par cas.

Avant de parler d'hérésie économique pour un ouvrage, il faut d'abord bien cerner le problème. Par exemple avant de supprimer les emplois des pêcheurs professionnels il faut bien mesurer l'enjeu et faire une étude globale.
De plus il n'y a pas de petits et de grands migrateurs, il y a des migrateurs. Chaque cas doit donc être étudié avec une méthode appropriée.

On ne peut pas prétendre qu'il n'y ait pas de solution et rejeter d'emblée la possibilité du système compensateur.

Néanmoins, on a déjà montré que, notamment en limite de l'aire d'expansion de certaines espèces, on peut imaginer des dispositions contractuelles avec le contractant qui dispose d'autres ouvrages situés sur le bassin.

Il ne croit pas qu'on puisse, dans un premier temps, parler de l'hérésie économique qui consisterait à construire des passes qui ne seraient pas efficaces. D'abord elles sont obligatoires en tant que dispositions annexes aux conditions d'environnement. II rappelle, d'ailleurs, que les contraintes d'environnement ont évolué par rapport aux dispositions existant il y a quelques années.

Il faut négocier cas par cas mais dans les aménagements importants et des ouvrages correspondants dans un bassin tel que celui de l'axe Loire-Allier, il faut insister sur l'étude globale préalable qui doit porter sur l'ensemble des aspects, notamment les aspects économiques liés à la pêche professionnelle et au

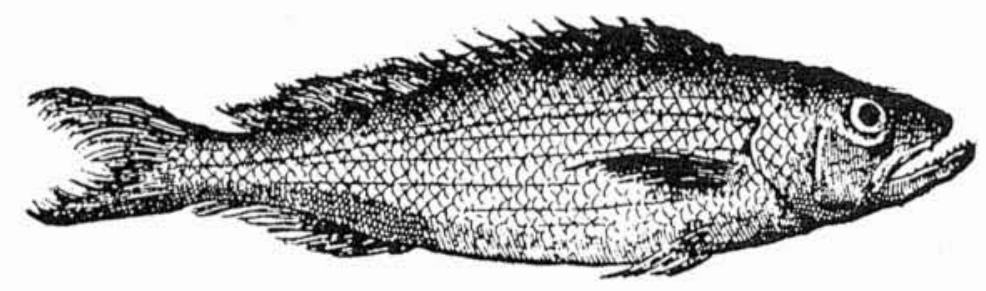

